\title{
Multihoming of Users to Access Points in WLANs: A Population Game Perspective
}

\author{
Srinivas Shakkottai, Eitan Altman, and Anurag Kumar
}

\begin{abstract}
We consider non-cooperative mobiles, each faced with the problem of which subset of WLANs access points (APs) to connect and multihome to, and how to split its traffic among them. Considering the many users regime, we obtain a potential game model and study its equilibrium. We obtain pricing for which the total throughput is maximized at equilibrium and study the convergence to equilibrium under various evolutionary dynamics. We also study the case where the Internet Service Provider (ISP) could charge prices greater than that of the cost price mechanism and show that even in this case multihoming is desirable.
\end{abstract}

Index Terms-Multihoming, IEEE 802.11 WLANs, game theory, population games, optimization.

\section{INTRODUCTION}

T HE IEEE 802.11 protocol is currently the standard for wireless LANs (WLANs). Quite often there is a choice of access points (APs) to which a mobile user could connect to. Users scan the wireless channels in order to find the AP of the highest signal strength and associate to it. They then transmit at different rates (called the PHY rate) based on the signal strength indicated. It has been observed [1] that all the connections in a single cell receive the same throughput, leading to inefficient channel use. The question arises as to whether it might be better for a user to split traffic among visible APs, which would provide diversity from the fact that signal strength in different cells might be different and the cells might be loaded differently.

Suppose we have a geographical region divided into cells as shown in Figure 1. Each cell would have an AP, each using a separate channel. This is possible in $802.11 \mathrm{~b}$ and $\mathrm{g}$ as they have three non-overlapping channels. Users may observe significant signal strengths from different APs. For instance, in Figure 1, users in region $A$ might be able to associate to cells $P, Q$ and $S$, whereas users in region $B$, might have no choice but to associate to cell $S$. It is possible for users with just a single wireless network interface card to associate to all the APs available to them, as has been shown in [2]. Users could then probabilistically divide their traffic among the different APs. The APs could charge a price for sending packets through

Manuscript received July 1, 2006; revised February 15, 2007. An earlier version of this paper was presented at IEEE INFOCOM, Barcelona, Spain, April 2006. Research supported in part by International Programs in Engineering at UIUC, Bionets Integrated Project on Autonomic Communications and the Indo-French Center for Promotion of Advanced Research IFCPAR Grant 2900-IT.

S. Shakkottai is at the University of Illinois at Urbana-Champaign (e-mail: sshakkot@uiuc.edu).

E. Altman is at INRIA Sophia Antipolis (e-mail: altman@sophia.inria.fr).

A. Kumar is at the Indian Institute of Science, Bangalore, India (e-mail: anurag@ece.iisc.ernet.in).

Digital Object Identifier 10.1109/JSAC.2007.070814.

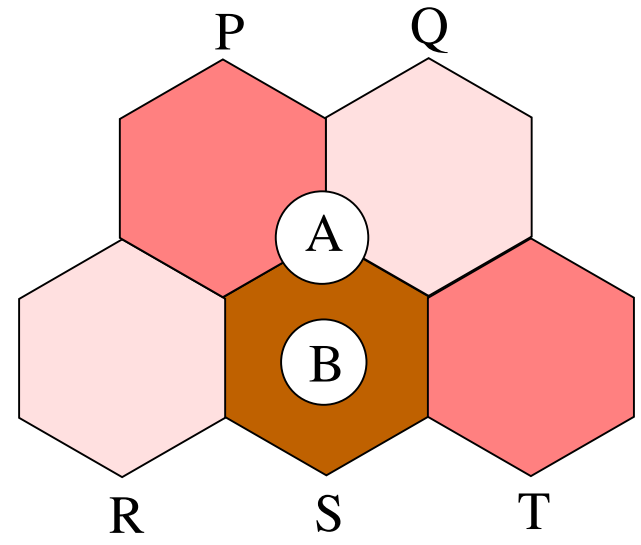

Fig. 1. Division of a geographical region into non-interfering cells using three independent channels, indicated by shading. Users might be able to connect to multiple APs.

them. The payoff that the users obtain is the difference of the throughput minus the price charged. Traffic splitting in the Internet among different Internet Service Providers (ISPs) is called multihoming [3] and we follow the same terminology for the WLAN case. Our objective in this paper is to design a decentralized pricing mechanism which has the property that even with selfish users trying to maximize their own payoffs, the system throughput is actually maximized.

\section{Related Work}

There has been much interest in understanding the behavior of wireless LANs. The question of why users using different PHY rates all obtain the same throughput was studied using simulation and experiments in [1]. In [4] the system was studied as a two-player game and results were presented on the inefficiency of the system as compared to the cooperative optimum. Bianchi [5] used fixed point analysis in order to provide an analytical framework for 802.11 WLANs. The results were generalized in [6], to provide expressions for the throughput of users with disparate frame sizes and PHY rates. The case of multiple TCP sessions in a WLAN environment was considered initially in [7] and later simplified and extended to the VoIP case as well in [8]. The analytical work has been further extended to IEEE 802.11e [9] where the relation between fixed point uniqueness and short term unfairness is also explored. The case of multiple APs being available is studied in [10], and the work focuses on fairness and load balancing in a cooperative scenario. In [11], the case of non-cooperative users who decide their frame size and PHY rate in order to maximize their individual throughputs 
is studied. Another paper on non-cooperative association is [12], which provides a study of the benefit of associating to the AP that would provide the best estimated link rate. Some results on cooperative association of users to different APs are provided in [13], and on the association of users with dual mode handsets to IEEE 802.11 APs and 3G base stations are provided in [14]. The idea of virtualizing a wireless card to use all available APs has been studied in [2], where the idea is to make one AP believe that the wireless card is asleep (which would cause the AP to buffer packets), while actually sending traffic to another AP. They also propose empirical methods by which the ratio of traffic associated to each AP could be chosen.

\section{Main Results}

We consider the existing system of $\mathrm{UDP}^{1}$ or $\mathrm{TCP}^{2}$ running over 802.11, with the added provision of multihoming. The multihoming protocol would decide the time spent associated with each of the APs, while the transport layer would be (multiple) UDP or TCP sessions. We do not insist on any particular multihoming protocol, but only make the assumption that it would be rational in the sense that it would allocate more time to APs that yield higher payoffs, and less time to those that do not. We assume that the time-scale at which users switch between the APs (a few times per second) is much greater than the time-scale at which the throughput of TCP or UDP over 802.11 converge to their average values (of the order of milliseconds). The multihoming protocol chooses the APs probabilistically. In the fluid model approximating the many users regime, the ratio of fluid split among APs gives the probabilities of associating with them.

In a WLAN since different users send at different PHY rates, they occupy the channel for different amounts of time. We propose a pricing mechanism in which users are charged based on their channel occupancy. We call this "cost price charging". We show that under this mechanism, when all users use either TCP or UDP along with rational multihoming protocols, the system is asymptotically stable. We also show that multihoming under the cost-price mechanism is efficient, i.e., the system throughput is maximized. Our analysis follows that of Sandholm [15], who provides results on the conditions required for a general class of dynamics to be asymptotically stable. He also shows that there is no loss of efficiency under strong symmetry conditions. Although, the conditions required do not hold in our case, we use similar techniques in order to prove stability and efficiency results.

Finally, we show that when an ISP charges differentiated prices above the cost price charge in the different APs, multihoming achieves at least the same profit as unihoming. So the ISP suffers no loss by allowing its customers to multihome. We further show that even in the case of differentiated pricing, the throughput of the system as a whole is at least that of unihoming, thus building a strong case for multihomed IEEE 802.11 wireless LANs.

\footnotetext{
${ }^{1}$ User Datagram Protocol

${ }^{2}$ Transmission Control Protocol
}

\section{Organization of the Paper}

The paper is organized as follows. In Section II we discuss the game theoretic concepts used. In Section III, we study models of rational dynamics that would be used for multihoming. In Section IV, we develop models for the transport layer protocols. We proceed in Section V, to study the system in a noncooperative scenario. We next study the efficiency of such an equilibrium in Section VI and show that the equilibrium is efficient. We study the economic impact of multihoming in Section VII. We conclude in Section VIII.

\section{BASIC IDEAS ON POPUlation GAMES}

We first introduce the game theoretic concepts that are used in this paper. Much of the discussion below may be found in [15]. A population game $F$, with $Q$ non-atomic classes of players is defined by a mass and a strategy set for each class and a payoff function for each strategy. By a non-atomic population, we mean that the contribution of each member of the population is infinitesimal. We denote the set of classes by $\mathcal{Q}=\{1, \ldots, Q\}$, where $Q \geq 1$. The class $q$ has mass $\hat{d}_{q}$. The set of strategies for class $q$ is denoted $\mathcal{S}_{q}=\left\{1, \ldots, S_{q}\right\}$. These strategies can be thought of as the actions that members of $q$ could possibly take. A particular strategy distribution is the way the class $q$ partitions itself into the different actions available, i.e., a strategy distribution for $q$ is vector of the form $y_{q}=\left\{y_{q}^{1}, y_{q}^{2}, \ldots y_{q}^{S_{q}}\right\}$, where $\sum_{i=1}^{S_{q}} y_{q}^{i}=\hat{d}_{q}$. The set of strategy distributions of a class $q \in \mathcal{Q}$, is denoted by $Y_{q}=\left\{y_{q} \in \mathbb{R}_{+}^{S_{q}}: \sum_{i=1}^{S_{q}} y_{q}^{i}=\hat{d}_{q}\right\}$. We denote the vector of strategy distributions being used by the entire population by $\mathbf{y}=\left\{y_{1}, y_{2}, \ldots, y_{Q}\right\}$, where $y_{q} \in Y_{q}$. The vector $\mathbf{y}$ can be thought of as the state of the system. Let the space of all strategy distributions be $\mathcal{Y}$. The marginal payoff function (per unit mass) obtained from strategy $i \in \mathcal{S}_{q}$ by users of class $q$, when the state of the system is $\mathbf{y}$ is denoted by $F_{q}^{i}(\mathbf{y}) \in \mathbb{R}$ and is assumed to be continuous and differentiable. Note that the payoffs to a strategy in class $q$ can depend on the strategy distribution within class $q$ itself. The total payoff to users of class $q$ is then given by $\sum_{i=1}^{S_{q}} F_{q}^{i}(\mathbf{y}) y_{q}^{i}$. Players may be cooperative or non-cooperative in behavior.

A commonly used concept in the context of infinitesimal players is the Wardrop equilibrium [16]. Consider any strategy distribution $y_{q}=\left[y_{q}^{1}, \ldots, y_{q}^{S_{q}}\right]$. There would be some elements which are non-zero and others which are zero. We call the strategies corresponding to the non-zero elements as the strategies used by class $q$.

Definition 1: A state $\hat{\mathbf{y}}$ is a Wardrop equilibrium if for any class $q \in \mathcal{Q}$, all strategies being used by the members of $q$ yield the same marginal payoff to each member of $q$, whereas the marginal payoff that would be obtained by members of $q$ is lower for all strategies not used by class $q$. Let $\hat{\mathcal{S}}_{q} \subset$ $\mathcal{S}_{q}$ be the set of all strategies used by class $q$ in a strategy distribution $\hat{\mathbf{y}}$. A Wardrop equilibrium $\hat{\mathbf{y}}$ is then characterized by the following relation:

$$
F_{q}^{s}(\hat{\mathbf{y}}) \geq F_{q}^{s^{\prime}}(\hat{\mathbf{y}}) \quad \forall s \in \hat{\mathcal{S}}_{q} \text { and } s^{\prime} \in \mathcal{S}_{q}
$$

If each class $q$ follows some dynamics, then would the stationary points be Wardrop equilibria? We present a result from [15], which is useful in this regard. We first need the following definition: 
Definition 2: The dynamics $\dot{\mathbf{y}}=\mathbf{V}(\mathbf{y})$ are said to be positively correlated (PC) if

$$
\sum_{q=1}^{Q} \sum_{i=1}^{S_{q}} F_{q}^{i}(\mathbf{y}) V_{q}^{i}(\mathbf{y})>0 \text { whenever } V(\mathbf{y}) \neq 0
$$

Result 1 If $\mathbf{V}(\mathbf{y})$ satisfies PC, all Wardrop equilibria of $F$ are the stationary points of $\dot{\mathbf{y}}=\mathbf{V}(\mathbf{y})$.

Potential games are a subclass of games that have a specific structure on the payoff functions that we study below.

Definition 3: We call $F$ a potential game if $\exists$ a $C^{1}$ function $\mathcal{T}: \mathcal{Y} \rightarrow \mathbb{R}$ such that $\frac{\partial \mathcal{T}}{\partial y_{q}^{i}}(\mathbf{y})=F_{q}^{i}(\mathbf{y})$ for all $\mathbf{y} \in \mathcal{Y}, i \in \mathcal{S}_{q}$ and $q \in \mathcal{Q}$.

The definition says that the rate of change of potential with mass of a population using a strategy is the payoff obtained per unit mass by that population for that strategy. We then immediately have that if $F$ is a potential game and $\mathbf{V}(\mathbf{y})$ is $\mathrm{PC}$, then the potential function $\mathcal{T}$ is a Lyapunov function for the system $\dot{\mathbf{y}}=\mathbf{V}(\mathbf{y})$. This means that all the stationary points of $\dot{\mathbf{y}}=\mathbf{V}(\mathbf{y})$ would be asymptotically stable. Thus, we have the following useful result.

Result 2 A potential game $F$, with dynamics $\mathbf{V}(\mathbf{y})$ that is $\mathrm{PC}$, has asymptotically stable stationary points.

In accordance with Result 1, the system state would converge to either a Wardrop equilibrium or a boundary point of the set $\mathcal{Y}$.

\section{System Dynamics: Multihoming Protocols}

As mentioned in the introduction, the population dynamics will be used to model the behavior of a multihoming protocol that decides how long to associate with each available AP. What would the characteristics of a good protocol be? The protocol must associate with APs that provide higher utilities for a longer time and with APs that provide lower utilities for a shorter time. Thus, at each time instant it must decide on the vector of probabilities of association with each available AP based on the current estimate of the utility obtained from each AP. We study two kinds of dynamics that fit the above characteristics. The first dynamics are called Replicator Dynamics [17]. The rate of increase of $\dot{y}_{q}^{s} / y_{q}^{s}$ of the strategy $s$ is a measure of its evolutionary success. We may express this success as the difference in fitness $F_{q}^{s}(\mathbf{y})$ of the strategy $s$ and the average fitness $\sum_{i=1}^{S_{q}} y_{q}^{i} F_{q}^{i}(\mathbf{y}) / \hat{d}_{q}$ of the class $q$. Then the dynamics used to describe changes in the mass of class $q$ playing strategy $s$ is given by

$$
\dot{y}_{q}^{s}=\mathbf{V}(\mathbf{y})=y_{q}^{s}\left(F_{q}^{s}(\mathbf{y})-\frac{1}{\hat{d}_{q}} \sum_{i=1}^{S_{q}} y_{q}^{i} F_{q}^{i}(\mathbf{y})\right) .
$$

Note that the dynamics take place within the set $\sum_{j=1}^{S_{q}} y_{q}^{j}=$ $\hat{d}_{q} \forall q \in\{1,2, \ldots, Q\}$, i.e., the total mass of each class remains fixed. This fact may be seen immediately by summing (1) over all strategies, yielding $\sum_{s=1}^{S_{q}} \dot{y}_{q}^{s}=0$.

Another commonly used model is called Brown-von Neumann-Nash (BNN) dynamics [18], which is somewhat more complex. Let,

$$
\gamma_{q}^{s}=\max \left\{F_{q}^{s}(\mathbf{y})-\frac{1}{\hat{d}_{q}} \sum_{i=1}^{S_{q}} y_{q}^{i} F_{q}^{i}(\mathbf{y}), 0\right\}
$$

denote the excess marginal payoff to strategy $s$ relative to the average payoff in its class. Then BNN dynamics are described by

$$
\dot{y}_{q}^{s}=\mathbf{V}(\mathbf{y})=\hat{d}_{q} \gamma_{q}^{s}-y_{q}^{s} \sum_{j=1}^{S_{q}} \gamma_{j}^{s},
$$

where the dynamics take place within the set $\sum_{j=1}^{S_{q}} y_{q}^{j}=$ $\hat{d}_{q} \forall q \in\{1,2, \ldots, Q\}$. Unlike replicator dynamics, it has the property of allowing extinct strategies to resurface, so that its stationary points are always Wardrop equilibria. It is not difficult to prove the following theorem.

Theorem 1: Both Replicator and BNN dynamics are positively correlated. Within a given problem, when each class of the population follows either replicator or BNN dynamics, the system is positively correlated.

The proof of the above theorem may be found in [15] or [19], which we do not repeat here due to space constraints. We next consider the transport layer over which the multihoming protocol would run.

\section{TRANSPORT LAYER MODELS}

Let there be $S$ independent APs, which do not interfere with each other by appropriate channel allocation. Let us denote $S_{q}$ as the number of APs available to users of class $q, L_{q}$ as the frame size, and $R_{q}^{i}$ is as PHY rate that a user of class $q$ would have if it connected to the $i$ th AP. We define a class $q$ of users as the set of all users that have access to the same APs and common values of $\left[L_{q}, R_{q}^{1}, R_{q}^{2}, \ldots, R_{q}^{S_{q}}\right]$. The class is used to model the fact that users in the same geographical location would face a similar set of circumstances. We assume that classes do not change under the time scale studied (of the order of seconds), which models real users who stay in a fixed location for minutes at a time. Let there be $d_{q}$ users of class $q$. Of these, assume a fraction $x_{q}^{s}$ is connected to AP $s$. The total number of users connected to AP $s$ is then $n^{s}=\sum_{q=1}^{Q} d_{q} x_{q}^{s}$. We define

$$
\alpha_{q}^{s} \triangleq \frac{d_{q} x_{q}^{s}}{n^{s}}=\frac{d_{q} x_{q}^{s}}{\sum_{i=1}^{Q} d_{i} x_{i}^{s}},
$$

which is understood to be zero if the denominator is zero. We wish to take the limit as $n^{s}$ becomes large simultaneously for all $s$ as a common parameter $n$ goes to infinity, thus keeping the fractions of each user class fixed as we scale $n$. Hence, we consider the following scaling:

$$
d_{q}=n \hat{d}_{q} .
$$

$n$ can be interpreted as the sum of all demand, i.e., $n=\sum_{q=1}^{Q} d_{q}$.

\section{Model for UDP sessions}

We construct a model based on expressions for UDP traffic obtained in [5], [6], [11]. We assume every user has infinitely 
many packets backlogged in its transmission buffer. Consider a particular AP $s$, and let $p$ be the exponential back-off multiplier, i.e., if $b_{z}$ is the mean back-off duration (in slots) at the $z$ th attempt for a frame then $b_{z}=p^{z} b_{0}$. Also let $T_{o}$ be defined as the transmission overhead in slots related to a frame transmission, which comprises of the SIFS/DIFS ${ }^{3}$, etc. and let $T_{c}$ be defined as the fixed overhead for an RTS collision in slots ${ }^{4}$. Also, assume that there are $n^{s}$ users associated with an AP of which $m_{q}^{s}$ users belong to class $q$. Denote by $\alpha_{q}^{s}=m_{q}^{s} / n^{s}$ the fraction of the users of class $q$ among all users in the cell, where $\alpha_{q}^{s}$ could be zero. Then proceeding as in Theorem VII.2 in [6], the total throughput of all users of class $q$ as $n^{s} \rightarrow \infty$ is given by

$$
\tau\left(\alpha_{q}^{s}\right)=\frac{\alpha_{q}^{s} L_{q}}{\kappa+\sum_{j=1}^{Q} \frac{\alpha_{j}^{s} L_{j}}{R_{j}^{s}}},
$$

where

$$
\kappa=\frac{p+T_{c}}{(p-1) \ln \left(\frac{p}{p-1}\right)}+T_{o}-T_{c}
$$

Using the fluid scaling described in (4)-(5) and defining $y_{q}^{s} \triangleq \hat{d}_{q} x_{q}^{s}, w_{q}^{s} \triangleq \frac{L_{q}}{R_{q}^{s}}$, the throughput received by the total mass of users of class $q$ connected to AP $s$ is

$$
T_{q}^{s}\left(\mathbf{y}^{s}\right) \triangleq \frac{L_{q}}{\kappa \sum_{j=1}^{Q} y_{j}^{s}+\sum_{j=1}^{Q} y_{j}^{s} w_{j}^{s}} .
$$

Note that the total mass of users of class $q$ is given by $\sum_{i=1}^{S_{q}} y_{q}^{i}=\hat{d}_{q}$.

\section{Model for TCP sessions}

We now consider the case in which several users are downloading large files via the AP to which they are associated under the control of TCP. The files are being downloaded from servers on the wired local area network, which is assumed to be very fast, and hence not a bottleneck. In such a system, the AP will only send data packets, while the users only send ACKs. The expressions for all users having identical PHY rates have been worked out in [7], [8]. We present a slightly modified version of the results, which apply to our case of dissimilar users. The analysis proceeds by identifying cycles during each of which the number of users with acks remains constant. As before, let us consider an AP $s$, with the fraction of users of class $q$ being $\alpha_{q}^{s}$. The total throughput of all users of class $q$ when the number of simultaneous TCP sessions is large is

$$
\tau\left(\alpha_{q}^{s}\right)=\alpha_{q}^{s} L_{q} \frac{\sum_{i=0}^{\infty} \pi_{i}\left(\frac{1}{i+1}\right)}{\sum_{i=0}^{\infty} \pi_{i} E_{i} X},
$$

where the stationary probability of $i$ contending users in a cycle $\pi_{i}=\frac{i+1}{i ! 2 e}, i \in\{0,1,2, \ldots\}$, and

$$
\begin{aligned}
E_{i} X & =\frac{P_{\text {idle }}(i) \delta}{1-P_{\text {idle }}(i)-P_{C}(i)}+\frac{P_{C}(i) T_{c}^{s}}{1-P_{\text {idle }}(i)-P_{C}(i)} \\
& +\frac{P_{\text {User }}(i) T_{U s e r}^{s}}{1-P_{\text {idle }}(i)-P_{C}(i)}+\frac{P_{\text {AP }}(i) T_{A P}^{s}}{1-P_{\text {idle }}(i)-P_{C}(i)}
\end{aligned}
$$

\footnotetext{
${ }^{3}$ Short Inter-Frame Space/Distributed Inter-Frame Space

${ }^{4}$ According to the IEEE 802.11 specifications $p=2$.
}

In the above expression $P_{i d l e}(i), P_{c}(i), P_{U s e r}(i)$ and $P_{A P}(i)$ are the probabilities of the channel remaining idle, of collision, of a user winning the channel and that the AP wins the channel respectively. Also, $\delta, T_{c}^{s}, T_{U s e r}^{s}$ and $T_{A P}^{s}$ are the system slot time, the average length of a cycle with a collision, the cycle length for a successful ack, and the cycle length for successful transmission of a data packet, respectively.

We associate each class of user in $s$ with the parameters $\left[L_{q}, T_{q(C)}^{s}, T_{q(A P)}^{s}, T_{q(\text { User })}^{s}\right]$, where $T_{q(C)}^{s}, T_{q(A P)}^{s}, T_{q(U s e r)}^{s}$ are the time spent in a collision, time taken for APs to transmit a packet, and the time taken for a user of class $q$ to transmit an ack to AP $s$. The expressions relating these quantities to the PHY rates can be found from [7] or [8]. We then have that if the user mass of class $q$ is denoted by $y_{q}^{s}$, then

$$
\begin{array}{r}
T_{C}^{s}=\frac{\sum_{i=1}^{Q} T_{i(C)}^{s} y_{i}^{s}}{\sum_{i=1}^{Q} y_{i}^{s}}, T_{U s e r}^{s}=\frac{\sum_{i=1}^{Q} T_{i(U s e r)}^{s} y_{i}^{s}}{\sum_{i=1}^{Q} y_{i}^{s}} \\
T_{A P}^{s}=\frac{\sum_{i=1}^{Q} T_{i(A P)}^{s} y_{i}^{s}}{\sum_{i=1}^{Q} y_{i}^{s}}
\end{array}
$$

Substituting the above into (9) and simplifying we get that the throughput per unit mass (in bits per second) obtained by users of class $q$ is

$$
T_{q}^{s}\left(\mathbf{y}^{s}\right)=\frac{L_{q}}{G\left(\mathbf{y}^{s}\right)}
$$

where

$$
\begin{aligned}
G\left(\mathbf{y}^{s}\right) & =\kappa_{1} \sum_{j=1}^{Q} y_{j}^{s}+\kappa_{2} \sum_{j=1}^{Q} y_{j}^{s} T_{j(C)}^{s} \\
& +\kappa_{3} \sum_{j=1}^{Q} y_{j}^{s} T_{j(\text { User })}^{s}+\kappa_{3} \sum_{j=1}^{Q} y_{j}^{s} T_{j(A P)}^{s},
\end{aligned}
$$

and

$$
\begin{aligned}
& \kappa_{1}= \frac{\sum_{i=0}^{\infty} \pi_{i} \frac{P_{\text {idle }}(i) \delta}{1-P_{\text {idle }}(i)-P_{C}(i)}}{\sum_{i=0}^{\infty} \pi_{i}\left(\frac{1}{i+1}\right)}, \\
& \kappa_{2}=\frac{\sum_{i=0}^{\infty} \pi_{i} \frac{P_{C}(i)}{1-P_{\text {idle }}(i)-P_{C}(i)}}{\sum_{i=0}^{\infty} \pi_{i}\left(\frac{1}{i+1}\right)}, \\
& \kappa_{3}=\frac{\sum_{i=0}^{\infty} \pi_{i} \frac{P_{U \text { ser }}(i)}{1-P_{\text {idle }}(i)-P_{C}(i)}}{\sum_{i=0}^{\infty} \pi_{i}\left(\frac{1}{i+1}\right)}, \\
& \kappa_{4}=\frac{\sum_{i=0}^{\infty} \pi_{i} \frac{P_{A P}(i)}{1-P_{\text {idle }}(i)-P_{C}(i)}}{\sum_{i=0}^{\infty} \pi_{i}\left(\frac{1}{i+1}\right)} .
\end{aligned}
$$

Note the similarity between (8) and (12). Both are of the general form

$$
T_{q}^{s}\left(\mathbf{y}^{s}\right)=\frac{\eta_{q}}{\sum_{j=1}^{Q} \mu_{j} y_{j}^{s}},
$$

with $\eta_{q}$ and $\mu_{q}$ being constants associated with class $q$. All the analysis in the future sections would apply to any utility function of the above form. We will focus on the expressions pertaining to the UDP as the transport protocol in the rest of the paper. 


\section{The Non-Cooperative Multihoming Problem}

The system we study consists of all users running UDP sessions, with a multihoming protocol riding on top that decides the duration of time (and hence user masses) spent associated with each available AP. As explained in Section III, we model the multihoming protocol using positively correlated dynamics. Also we assume that the time-scale of association is such that the sessions attain their equilibrium values (given in (6) in a short time. We thus have a system with $Q$ classes of users, who partition themselves among the different actions available to them in the way that is optimal for themselves. We denote this non-cooperative population game by $F$. We first study the costs and payoffs associated with the system, which are measured in units of throughput. As before, let the $\mathbf{y}$ denote the strategy profile of the system. The total system throughput is given by

$$
\mathcal{T}(\mathbf{y}) \triangleq \sum_{k=1}^{S} \sum_{i=1}^{Q} \tau_{i}^{k}(\mathbf{y})=\sum_{k=1}^{S} \sum_{i=1}^{Q} y_{i}^{k} T_{i}^{k}(\mathbf{y})
$$

All users contribution to this throughput is not the same. Given a time interval, there are some users taking only a small time share and others who take a large time share, which depends on the PHY rate and the frame size that he or she uses. From (6), we calculate that the channel "occupancy factor" per unit mass for UDP is given by

$$
\delta_{q}^{s}(\mathbf{y}) \triangleq \frac{\kappa+w_{q}^{s}}{\kappa \sum_{j=1}^{Q} y_{j}^{s}+\sum_{j=1}^{Q} y_{j}^{s} w_{j}^{s}} .
$$

It gives the ratio of time occupied by users of class $q$ to the total amount of time occupied by all users. Thus, a lower occupancy means that a class is being more efficient. The occupancy can be thought of as the externality that the user exerts on the others. In terms of throughput, the cost of externalities of users of class $q$ in cell $s$ is the occupancy times the total throughput of all users in the cell, i.e., the cost of a unit mass of users of class $q$ is

$$
C_{q}^{s}(\mathbf{y}) \triangleq \delta_{q}^{s}(\mathbf{y}) \sum_{i=1}^{Q} \tau_{i}^{s}(\mathbf{y})
$$

Suppose that the AP charges the above price to users of class $q$. We call this pricing mechanism as "cost-price" since revenue obtained in a cell is identical to the total throughput in the cell. We can see this fact by considering the total revenue generated in a cell, obtained from (17):

$$
\begin{aligned}
\sum_{i=1}^{Q} C_{i}^{s}(\mathbf{y}) y_{i}^{s} & =\sum_{i=1}^{Q}\left(\delta_{q}^{s}(\mathbf{y}) \sum_{j=1}^{Q} \tau_{j}^{s}(\mathbf{y}) y_{i}^{s}\right) \\
& =\sum_{j=1}^{Q} \tau_{j}^{s}(\mathbf{y}) \sum_{i=1}^{Q} \delta_{q}^{s}(\mathbf{y}) y_{i}^{s} \\
& =\sum_{j=1}^{Q} \tau_{j}^{s}(\mathbf{y}) \sum_{i=1}^{Q} \frac{\left(\kappa+w_{q}^{s}\right) y_{i}^{s}}{\kappa \sum_{j=1}^{Q} y_{j}^{s}+\sum_{j=1}^{Q} y_{j}^{s} w_{j}^{s}} \\
& =\sum_{j=1}^{Q} \tau_{j}^{s}(\mathbf{y}),
\end{aligned}
$$

\begin{tabular}{|c|c|}
\hline$\overline{\mathcal{Q}}$ & Set of classes of players. \\
\hline$Q$ & Number of player classes (cardinality of $\mathcal{Q}$ ). \\
\hline$q$ & A particular class of players $(q \in \mathcal{Q})$ \\
\hline$\hat{d}_{q}$ & Mass of players belonging to class $q$. \\
\hline $\mathcal{S}_{q}$ & $\begin{array}{l}\text { Set of strategies of class } q \text {, also the set of APs that users of } \\
\text { class } q \text { can connect to. }\end{array}$ \\
\hline$y_{q}$ & $\begin{array}{l}\text { Strategy distribution of class } q \text {, also the vector of the masses } \\
\text { of players in class } q \text { associated with each available AP. }\end{array}$ \\
\hline $\mathbf{y}$ & The vector of all $y_{q}$ 's, referred to as the system state. \\
\hline$y_{q}^{k}$ & $\begin{array}{l}\text { The mass of players of class } q \text { that play strategy } k \text {, also } \\
\text { the mass of users of class } q \text { associated with AP } k \text {. }\end{array}$ \\
\hline $\mathcal{Y}$ & The set of all possible strategy distributions. \\
\hline$\tau_{q}^{s}(\mathbf{y})$ & $\begin{array}{l}\text { The total throughput that users of class } q \text { obtain from AP } \\
s \in \mathcal{S}_{q} \text {, when the state of the system is } \mathbf{y} \text {. }\end{array}$ \\
\hline$T_{q}^{s}(\mathbf{y})$ & $\begin{array}{l}\text { The throughput per unit mass that users of class } q \text { obtain } \\
\text { from AP } s \in \mathcal{S}_{q} \text {, when the state of the system is } \mathbf{y} \text {. }\end{array}$ \\
\hline$C_{q}^{s}(\mathbf{y})$ & $\begin{array}{l}\text { The price charged per unit mass by AP } s \in \mathcal{S}_{q} \text { to users of } \\
\text { class } q \text {, when the state of the system is } \mathbf{y} \text {. }\end{array}$ \\
\hline$F_{q}^{i}(\mathbf{y})$ & $\begin{array}{l}\text { The marginal payoff per unit mass from strategy } i \in \mathcal{S}_{q} \text { by } \\
\text { users of class } q \text {, when the state of the system is } \mathbf{y} \text {, also the } \\
\text { throughput minus price per unit mass. }\end{array}$ \\
\hline $\mathcal{T}(\mathbf{y})$ & $\begin{array}{l}\text { Potential function, which turns out to be the total system } \\
\text { throughput with system state } \mathbf{y} \text {. }\end{array}$ \\
\hline
\end{tabular}

TABLE I

SUMMARY OF NOTATION USED IN POPULATION GAMES AND MULTIHOMING MODEL

which is the total throughput in the cell. We have used the definition of occupancy (16) in the above derivation. User payoff is the throughput minus the price paid, and hence the payoff function per unit mass for users of class $q$ in cell $s$ is

$$
F_{q}^{s}(\mathbf{y}) \triangleq T_{q}^{s}(\mathbf{y})-C_{q}^{s}(\mathbf{y})
$$

The above tells a user the value of associating to a particular AP. We show below that under the above pricing scheme, the total system throughput is a potential function for the game. The main notation used is shown in Table I.

Theorem 2: The system throughput

$$
\mathcal{T}(\mathbf{y})=\sum_{k=1}^{S} \sum_{i=1}^{Q} y_{i}^{k} T_{i}^{k}(\mathbf{y})
$$

where $y_{i}^{j}=0$ if AP $j$ is not available to user $i$ is a potential function for the game $F$ with cost-price charging.

Proof: We have

$$
\begin{aligned}
\frac{\partial \mathcal{T}(\mathbf{y})}{\partial y_{q}^{s}}= & \frac{\partial}{\partial y_{q}^{s}} \sum_{k=1}^{S} \sum_{i=1}^{Q} y_{i}^{k} T_{i}^{k}(\mathbf{y}) \\
= & \frac{\partial}{\partial y_{q}^{s}} \sum_{k=1}^{S} \sum_{i=1}^{Q} \frac{y_{i}^{k} L_{i}}{\kappa \sum_{j=1}^{Q} y_{j}^{k}+\sum_{j=1}^{Q} y_{j}^{k} w_{j}^{k}} \\
= & \frac{L_{q}}{\kappa \sum_{j=1}^{Q} y_{j}^{k}+\sum_{j=1}^{Q} y_{j}^{k} w_{j}^{k}} \\
& -\frac{\left(\kappa+w_{q}^{s}\right) \sum_{i=1}^{Q} y_{i}^{s} L_{i}^{s}}{\left(\kappa \sum_{j=1}^{Q} y_{j}^{k}+\sum_{j=1}^{Q} y_{j}^{k} w_{j}^{k}\right)^{2}} \\
= & T_{q}^{s}(\mathbf{y})-\delta_{q}^{s}(\mathbf{y}) \sum_{i=1}^{Q} \tau_{i}^{s}(\mathbf{y})=F_{q}^{s}(\mathbf{y}),
\end{aligned}
$$

which means that $\mathcal{T}(\mathbf{y})$ satisfies the definition of a potential function. 
We have thus shown that the conditions required for Result 2 to hold are satisfied, and hence the stationary points of the dynamics are asymptotically stable ${ }^{5}$. From the form of the potential function, we expect that the stationary point actually maximizes the throughput.

\section{THE PRICE OF ANARCHY}

We would like to know what effect selfish multihoming has on the efficiency of the system. In most work on selfish routing (such as [20]), it is found that the Wardrop equilibrium is inefficient, and this inefficiency is referred to as the price of anarchy. In [15] it is shown that under strong symmetry conditions on the payoffs and potential function, efficiency can be achieved in a potential game. Our scenario does not admit such strong conditions due to the structure of the system considered. However, we have just seen that the total throughput acts as a potential function for the system. Below we provide a characterization of the stationary point and show that it is efficient.

For both the replicator (1) as well as the BNN dynamics (3), we have that $\dot{y}_{q}^{s}=0$, implies that either

$$
F_{q}^{s}(\hat{\mathbf{y}})=\frac{1}{\hat{d}_{q}} \sum_{i=1}^{S_{q}} \hat{y}_{q}^{i} F_{q}^{i}(\hat{\mathbf{y}}) \quad \text { or } \quad \hat{y}_{q}^{s}=0,
$$

where we use $\hat{\mathbf{y}}$ to denote a stationary point. The above relations mean that users of class $q$ would get identical payoffs in all APs that they use at equilibrium. The potential payoff in all the APs not in use would be lower than this value. As mentioned earlier, the set of stationary points contains the set of Wardrop equilibria, and in the case of BNN dynamics, they are the same.

Now, consider the stationary point again. We define $\hat{F}_{q} \triangleq$ $\frac{1}{\hat{d}_{q}} \sum_{i=1}^{S_{q}} \hat{y}_{q}^{i} F_{q}^{i}(\hat{\mathbf{y}})$. Then the stationary point conditions look like Kuhn-Tucker first order conditions of an optimization problem. Let us identify the Lagrange dual function associated with the above expressions. It is seen that the minimization problem

$\min _{\lambda} \max _{\mathbf{y}}\left(\sum_{k=1}^{S} \sum_{i=1}^{Q} y_{i}^{k} T_{i}^{k}(\mathbf{y})-\sum_{i=1}^{Q} \lambda_{i}\left(\sum_{j=1}^{S_{i}} y_{i}^{j}-\hat{d}_{i}\right)\right)$,

yields (20) as the Kuhn-Tucker first order conditions with $\hat{F}_{i}=$ $\lambda_{i} \forall i \in\{1,2, \ldots, Q\}$ We then have the following theorem:

Theorem 3: The equilibrium of the non-cooperative game $F$ is identical to the solution of the constrained optimization problem

$$
\max _{\mathbf{y}}\left(\sum_{k=1}^{S} \sum_{i=1}^{Q} y_{i}^{k} T_{i}^{k}(\mathbf{y})\right)
$$

subject to the constraints

$$
\sum_{j=1}^{S_{i}} y_{i}^{j}=\hat{d}_{i} \quad \forall i \in\{1,2, \ldots, Q\}
$$

and $y_{i}^{j}=0$ if AP $j$ is not available to users of class $i$.

\footnotetext{
${ }^{5}$ Note that the potential function could have non-unique maxima, and the system state would converge to one of these.
}

Proof: From the above discussion we have that the noncooperative game $F$ converges to the solution of the Lagrange dual problem (21). Call the solution obtained as $\mathcal{T}(\hat{\mathbf{y}})$. Also, call the solution to the primal problem (22) as $\mathcal{T}\left(\mathbf{y}^{\star}\right)$. Now, the expression in (22) is not concave and there could exist multiple maxima. There could also be a duality gap between the primal and dual problem, i.e., $\mathcal{T}(\hat{\mathbf{y}}) \geq \mathcal{T}\left(\mathbf{y}^{\star}\right)$. But it is physically impossible for the system to converge to a state whose throughput is greater than the maximum possible, i.e., $\mathcal{T}(\hat{\mathbf{y}})=\mathcal{T}\left(\mathbf{y}^{\star}\right)$

We have shown that multihoming users with dissimilar selfish dynamics being charged the cost price of their occupancy actually optimize the system throughput. In the language of the above literature, the result states that anarchy is free! Our result is somewhat reminiscent of a result in [21], which states that the stability region of a network is increased by allowing multi-path routing and congestion control. But our formulation is unique to wireless LAN case. In the above discussion, we have assumed that there are no feedback delays in the system. The time for convergence is of the same order as the time of convergence of TCP to its equilibrium rate, i.e., of the order of milliseconds.

\section{Example}

As mentioned in the literature review, the models of UDP and TCP performance in WLANs that we use have been independently verified by experiments, while the multihoming idea has been implemented already. Here we show, using a simple Simulink example, the intuition behind why a positively correlated multihoming protocol would maximize the system throughput. Consider the scenario where there are two classes of users with the same two APs available to them. Their values of frame size are both equal to unity. The values of $\kappa+L / C$ are different - class 1 users have parameters $[2,1.5]$, while class 2 users have parameters $[1,5]$ in the two APs. The users are assumed to be downloading large files. Each user starts multiple TCP sessions, with one session corresponding to each visible AP. Initially, the file is split up into pieces with one piece to a TCP session. As the sessions finish transmitting their associated piece, the remaining amount is again split up among the sessions so as to keep them all saturated until the end. The multihoming protocol must decide how long to spend associated with each visible AP. In our simulation we assume that users use replicator dynamics as the multihoming protocol. Under this scenario the throughput is maximized if all users of class 1 use AP 1 while all users of class 2 use AP 2, which is a degenerate case of multihoming. Under the cost price mechanism, class 1 would have a negative payoff for using AP 2 and vice-versa for class 2. A rational multihoming protocol would thus cause class 1 to shift to AP 1 , and class 2 to shift to AP 2 causing throughput maximization. The throughput would then be 0.5 in AP 1 and 0.2 in AP 2. We illustrate that the throughputs do indeed converge to these values in Figure 2.

\section{ECONOMICS OF Multihoming}

We now consider a market model under which an ISP which owns all the APs can charge more than the cost price. For 


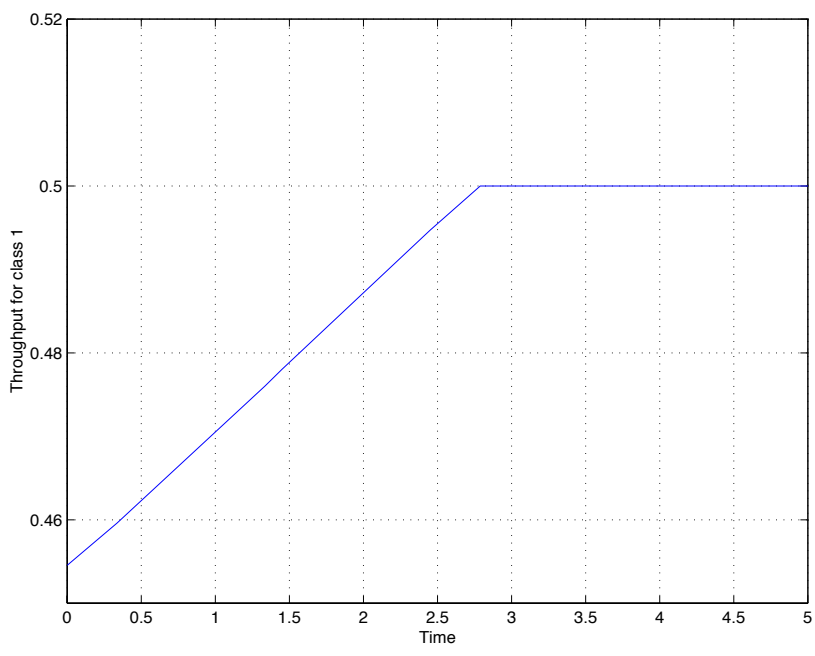

(a)

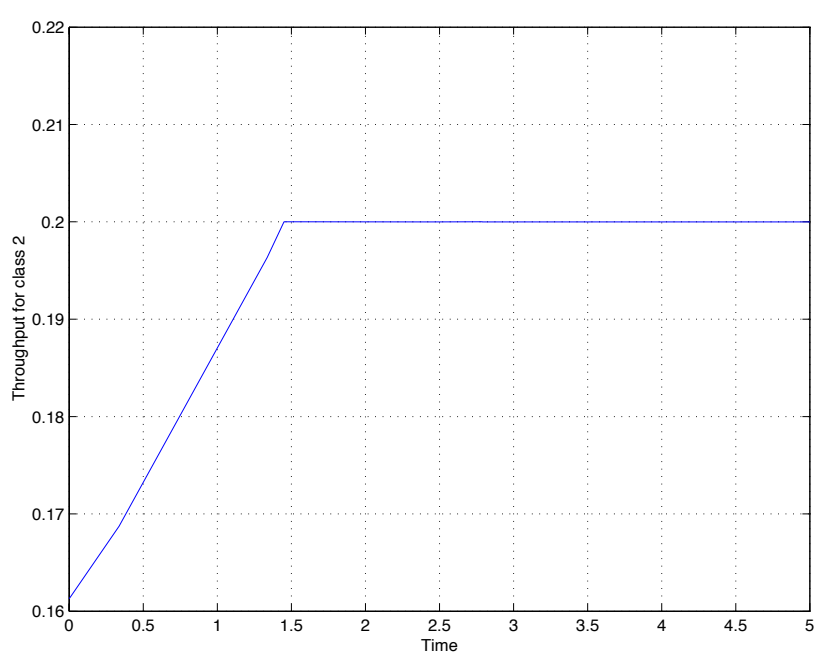

(b)

Fig. 2. Illustrating the convergence of throughputs to the stationary points.

example, in a building such as an airport, a single ISP would own all the APs. We assume that the potential mass of users in each class $q$ is a fixed value denoted by $\hat{d}_{q}$. In practice one would expect a variation of user masses over the course of a day as they move around. However, we can think of this as the average value. The actual mass of users in the system would depend on the prices charged. Let the subscription price per unit mass charged in AP $s$ to all users be denoted by $P^{s}$, where for generality we assume that the subscription price charged at different APs can be different. In addition to the subscription price, we assume that users are also charged the cost price of traffic described in the previous sections. The price vector $\mathbf{P} \triangleq\left[P^{1}, P^{2}, \ldots, P^{S}\right]$ would determine both the mass of users in the system, and the way this mass gets partitioned between the different APs. In our model, each class $q$ is associated with a threshold value $\Lambda_{q}$. Users of class $q$ would connect to an AP $s$ if $P^{s} \leq \Lambda_{q}$. The model is similar to the ones studied in [22], [23]. Once users connect to an AP, the throughput that they obtain is determined by (8). The payoff per unit mass is then

$$
F_{q}^{s}(\mathbf{y}) \triangleq T_{q}^{s}(\mathbf{y})-C_{q}^{s}(\mathbf{y})-P^{s}
$$

The ISP would like to maximize the profit regardless of the actual throughput of the system. The profit that the ISP makes is the difference between the total revenue and the cost (which we have assumed is equal to the actual throughput). Hence, the profit function of the ISP is

$$
\rho_{\text {multi }}(\mathbf{P}) \triangleq \sum_{j=1}^{S} P^{j} \sum_{i=1}^{Q} y_{i}^{j},
$$

where $y_{i}^{j}=0$ if users of class $i$ do not connect to AP $j$. We would like to know if the ISP has an incentive to allow multihoming.

\section{Effect on Profit}

We have to compare the profit when multihoming is an option and when it is not. We make the assumption that if multihoming were not allowed, users would connect only to (available) APs that display the lowest price. We denote this lowest price available to users of class $i$ by $P_{\min (i)}$. Then we have that the mass of users of class $i$ connecting to AP $k$, under a given pricing vector $\mathbf{P}$ is such that $y_{i}^{k}=0$ if $P^{k} \neq P_{\min (i)}$. Thus the profit function under unihoming is

$$
\rho_{\text {uni }}(\mathbf{P}) \triangleq \sum_{i=1}^{Q} P_{\min (i)} \hat{d}_{i},
$$

where $\hat{d}_{i}=0$ if $P_{\min (i)}>\Lambda_{i}$. We are now ready to compare the two. We have the following theorem:

Theorem 4: For the same price vector $\mathbf{P}, \rho_{\text {multi }}(\mathbf{P}) \geq$ $\rho_{\text {uni }}(\mathbf{P})$

Proof: The proof is straightforward once we realize that under a given price vector, the total user mass in the system is the same. Thus, we have

$$
\begin{aligned}
\rho_{\text {multi }}(\mathbf{P}) & =\sum_{j=1}^{S} P^{j} \sum_{i=1}^{Q} y_{i}^{j} \\
& \geq \sum_{j=1}^{S} \sum_{i=1}^{Q} P_{\min (i)} y_{i}^{j}=\sum_{i=1}^{Q} P_{\min (i)} \sum_{j=1}^{S} y_{i}^{j} \\
& =\sum_{i=1}^{Q} P_{\min (i)} \hat{d}_{i}=\rho_{\text {uni }}(\mathbf{P})
\end{aligned}
$$

and we are done.

The result means any profit achievable when an ISP allows unihoming can be met or exceeded by allowing multihoming.

\section{Effect on Throughput}

We now understand what effect multihoming has on the throughput of a system given a price vector $\mathbf{P}$. As before, we study the system as a population game with dynamics that are PC.

Theorem 5: The function

$$
\mathcal{T}_{\text {multi }}(\mathbf{y}) \triangleq \sum_{k=1}^{S} \sum_{i=1}^{Q} y_{i}^{k}\left(T_{i}^{k}(\mathbf{y})-P^{k}\right) .
$$

where $y_{i}^{j}=0$ if either AP $j$ is not available to user $i$ or $P^{j}>\Lambda_{i}$ is a potential function for the game $F$. 
Proof: The proof is identical to that of Theorem 2 and is omitted.

As before we have a characterization of the equilibrium point as follows:

Theorem 6: The equilibrium of the non-cooperative game $F$ is the solution of the constrained optimization problem

$$
\max _{\mathbf{y}}\left(\sum_{k=1}^{S} \sum_{i=1}^{Q} y_{i}^{k}\left(T_{i}^{k}(\mathbf{y})-P^{k}\right)\right)
$$

subject to the constraints

$$
\sum_{j=1}^{S_{i}} y_{i}^{j}=\hat{d}_{i} \quad \forall i \in\{1,2, \ldots, Q\}
$$

and $y_{i}^{j}=0$ if $\mathrm{AP} j$ is not available to users of class $i$ or $P^{j}>\Lambda_{i}$.

Proof: The proof is identical to Theorem 3.

We now assume that the users are not allowed to multihome, and hence choose one of the APs displaying the lowest price. Let the AP that users of class $q$ select be $\chi_{q}$. Then the equivalent of $\mathcal{T}_{\text {multi }}$ is given as

$$
\mathcal{T}_{\text {uni }} \triangleq \sum_{k=1}^{S} \sum_{i=1}^{Q} y_{i}^{k}\left(T_{i}^{k}(\mathbf{y})-P^{k}\right),
$$

where $y_{i}^{k}=\hat{d}_{i}$ if $k=\chi_{i}$, and 0 else. Clearly $\mathcal{T}_{\text {multi }} \geq \mathcal{T}_{\text {uni }}$. We then have the following theorem on the throughputs in the two cases.

Theorem 7: Given a price vector $\mathbf{P}$, the system throughput when multihoming is permitted is at least that of when it is not.

Proof: Denote the equilibrium state when multihoming by $\hat{\mathbf{y}}$ and the state when unihoming by $\mathbf{y}^{\star}$. We have $\mathcal{T}_{\text {multi }} \geq$ $\mathcal{T}_{\text {uni }}$

$$
\begin{aligned}
\Rightarrow & \sum_{k=1}^{S} \sum_{i=1}^{Q} \hat{y}_{i}^{k}\left(T_{i}^{k}(\hat{\mathbf{y}})-P^{k}\right) \geq \sum_{k=1}^{S} \sum_{i=1}^{Q} y_{i}^{k \star}\left(T_{i}^{k}\left(\mathbf{y}^{\star}\right)-P^{k}\right) \\
\Rightarrow & \sum_{k=1}^{S} \sum_{i=1}^{Q} \hat{y}_{i}^{k} T_{i}^{k}(\hat{\mathbf{y}})-\rho_{\text {mult } i}(\mathbf{P}) \\
& \geq \sum_{k=1}^{S} \sum_{i=1}^{Q} y_{i}^{k \star} T_{i}^{k}\left(\mathbf{y}^{\star}\right)-\rho_{\text {uni }}(\mathbf{P}) \\
\Rightarrow & \sum_{k=1}^{S} \sum_{i=1}^{Q} \hat{y}_{i}^{k} T_{i}^{k}(\hat{\mathbf{y}}) \geq \sum_{k=1}^{S} \sum_{i=1}^{Q} y_{i}^{k \star} T_{i}^{k}\left(\mathbf{y}^{\star}\right),
\end{aligned}
$$

since from Theorem 4 we have $\rho_{\text {multi }}(\mathbf{P}) \geq \rho_{\text {uni }}(\mathbf{P})$ (and they are non-negative). Hence the proof.

Thus multihoming would do at least as well as unihoming in terms of throughput as well. It is straightforward to show that the profit is always bounded as the user masses split within the compact set $\sum_{j=1}^{S_{q}} y_{q}^{j} \leq \hat{d}_{q}$. The vector $\mathbf{P}$ for which the profit is maximum need not be unique. Among all the maximizing $\mathbf{P}$ vectors, the ISP could choose the one that ensures the highest throughput of the system.

\section{CONCLUSION}

In this paper we have sought to make a convincing case for an ISP to allow multihoming in its IEEE 802.11 WLANs. In the scenario considered, there would be a large number of users and multiple APs seen by each user. We constructed a fluid model of user populations in a WLAN and understood how their throughputs varied with movement of user masses. We showed that users charged by a simple mechanism, using selfish dynamics would actually maximize the system throughput when allowed the option of multihoming. We also studied the economics of multihoming as seen by the ISP and showed that there is no loss of profit or throughput when users are allowed to multihome. A possible future extension is the study of competition between different ISPs that might own different subsets of APs.

\section{REFERENCES}

[1] M. Heusse, F. Rousseau, G. Berger-Sabbatel, and A. Duda, "Performance Anomaly of 802.11b," in Proceedings of IEEE INFOCOM 2003, San Fransisco, CA, March 2003.

[2] R. Chandra, P. Bahl, and P. Bahl, "MultiNet: Connecting to Multiple IEEE 802.11 Networks Using a Single Wireless Card," in Proceedings of IEEE INFOCOM 2004, Hong Kong, March 2004.

[3] A. Akella, J. Pang, B. Maggs, S. Seshan, and A. Shaikh, "A Comparison of Overlay Routing and Multihoming Route Control," in Proceedings of the ACM SIGCOMM, Portland, Oregon, February 2004.

[4] G. Tan and J. Guttag, "The 802.11 MAC Protocol Leads to Inefficient Equilibria," in Proceedings of IEEE INFOCOM'05, Miami, FL, March 2005.

[5] G. Bianchi, "Performance analysis of the IEEE 802.11 distributed coordination function," IEEE Journal on Selected Areas in Communications, vol. 18, pp. 535-547, March 2000.

[6] A. Kumar, E. Altman, D. Miorandi, and M. Goyal, "New insights from a fixed point analysis of single cell IEEE 802.11 WLANs," in Proceedings of IEEE INFOCOM 2005, Miami, FL, March 2005.

[7] R. Bruno, M. Conti, and E. Gregori, "Throughput analysis of TCP Clients in Wi-Fi Hot Spot Networks," in Networking 2004, Athens, Greece, May 2004.

[8] G. Kuriakose, S. Harsha, A. Kumar, and V. Sharma, "Analytical Models for Capacity Estimation of IEEE 802.11 WLANs using DCF for Internet Applications," Submitted.

[9] R. Venkatesh, A. Kumar, and E. Altman, "Fixed point analysis of single cell IEEE 802.11e WLANs: uniqueness, multistability and throughput differentiation," in Proceedings of ACM SIGMETRICS 2005, Banff, Canada, June 2005.

[10] Y. Bejerano, S.-J. Han, and L. E. Li, "Fairness and load balancing in wireless LANs using association control," in Proceedings of ACM Mobicom, Philadelphia, PA, September 2004.

[11] E. Altman, A. Kumar, D. Kumar, and R. Venkatesh, "Cooperative and non-coooperative control in IEEE 802.11 WLANs," in Proceedings of the International Teletraffic Congress, Beijing, August 2005.

[12] G. Athanasiou, T. Korakis, O. Ercetin, and L. Tassiulas, "Dynamic Cross-Layer Association in 802.11-based Mesh Networks," in Proceedings of IEEE INFOCOM 2007 (to appear), Anchorage, Alaska, May 2007.

[13] A. Kumar and V. Kumar, "Optimal Association of Stations and APs in an IEEE 802.11 WLAN," in Proceedings of the National Conference on Communications (NCC), IIT Kharagpur, January 2005.

[14] K. Premkumar and A. Kumar, "Optimal association of mobile wireless devices with a wlan-3g access network," in Proceedings of IEEE ICC 2006, Istanbul, June 2006.

[15] W. H. Sandholm, "Potential Games with Continuous Player Sets," Journal of Economic Theory, vol. 97, pp. 81-108, January 2001.

[16] J. G. Wardrop, "Some theoretical aspects of road traffic research," in Proceedings of the Institute of Civil Engineers, vol. 1, 1952, pp. 325378.

[17] J. Hofbauer and K. Sigmund, Evolutionary Games and Population Dynamics. Cambridge University Press, 1998.

[18] G. W. Brown and J. von Neumann, "Solution of games by differential equations," Contributions to the Theory of Games I, Annals of Mathematical Studies, vol. 24, 1950. 
[19] S. Shakkottai, E. Altman, and A. Kumar, "The Case for Non-cooperative Multihoming of Users to Access Points in IEEE 802.11 WLANs," in Proceedings of IEEE INFOCOM 2006, Barcelona, Spain, April 2006.

[20] T. Roughgarden and E. Tardos, "How bad is selfish routing?" in IEEE Symposium on Foundations of Computer Science, 2000, pp. 93-102.

[21] H. Han, S. Shakkottai, C. V. Hollot, R. Srikant, and D. Towsley, "Overlay TCP for Multi-Path Routing and Congestion Control," to appear in IEEE/ACM Trans. Networking.

[22] R. E. Azouzi, E. Altman, and L. Wynter, "Telecommunications Network Equilibrium with Price and Quality-of-Service Characteristics," in Proceedings of the International Teletraffic Congress, Berlin, September 2003.

[23] S. Shakkottai and R. Srikant, "Economics of Network Pricing With Multiple ISPs," in Proceedings of IEEE INFOCOM 2005, Miami, FL, March 2005.

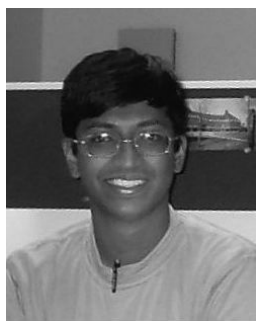

Srinivas Shakkottai received the Bachelor of Engineering degree in electronics and communication engineering from the Bangalore University, India, in 2001 and the M.S. degree in electrical engineering from the University of Illinois at Urbana-Champaign in 2003. He is currently pursuing a Ph.D. degree in the Department of Electrical and Computer Engineering at the University of Illinois at UrbanaChampaign.

His research interests include the design and analysis of peer-to-peer systems, pricing approaches to resource allocation in fixed and wireless networks, game theory, congestion control in the Internet and measurement and analysis of Internet data.

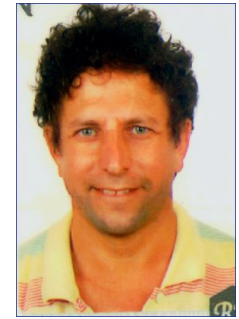

Eitan Altman received the B.Sc. degree in electrical engineering (1984), the B.A. degree in physics (1984) and the Ph.D. degree in electrical engineering (1990), all from the Technion-Israel Institute, Haifa. In (1990) he further received his B.Mus. degree in music composition in Tel-Aviv University. Since 1990, he has been with INRIA (National research institute in informatics and control) in Sophia-Antipolis, France. His current research interests include performance evaluation and control of telecommunication networks and in particular congestion control, wireless communications and networking games. He is in the editorial board of several scientific journals: JEDC, COMNET, DEDS and WICON. He has been the (co)chairman of the program committee of several international conferences and workshops on game theory, networking games and mobile networks.

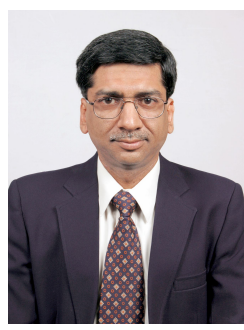

Anurag Kumar received the B.Tech. degree from IIT Kanpur and a PhD at Cornell University, both in EE. He was with Bell Labs, Holmdel, for over 6 years. He is now a Professor and Chair in the ECE Department at the Indian Institute of Science (IISc), Bangalore. His area of research is communication networking, and he has recently focused primarily on wireless networking. He is a Fellow of the IEEE, of the Indian National Science Academy (INSA), and of the Indian National Academy of Engineering (INAE). He is an associate editor of IEEE Transactions on Networking, and of IEEE Communications Surveys and Tutorials. $\mathrm{He}$ is a coauthor of the advanced text-book "Communication Networking: An Analytical Approach," by Kumar, Majunath and Kuri, published by MorganKaufman/Elsevier. 